\title{
Acoustic Frequency Optimization for Underwater Wireless Sensor Network
}

\author{
Emad Felemban \\ Department of Computer Engineering \\ Umm Al-Qura University \\ Makkah, Saudi Arabia
}

\begin{abstract}
In recent years, research in Underwater Wireless Sensor Network (UWSN) was the interest of many research groups as it can be used for many important applications such as disaster management, marine environment monitoring, fish farming, and military surveillance. There are many challenges in underwater acoustic communication: strong signal attenuation, limited bandwidth, long propagation delay, high transmission loss, and energy consumption. In this paper, we present a simple flow of mathematical models for the underwater acoustic channel for the underwater acoustic communication channel. We also investigate the influence of different parameters governing the communication channel's performance, such as temperature and wind speed. We also show the importance of selecting the optimal communication frequency to increase communication SNR. We implemented the mathematical model in MATLAB and made it available online for other researchers. We found out that selecting the optimal frequency is very crucial when wind speed is high.
\end{abstract}

Keywords-Underwater Wireless Sensor Network (UWSN); acoustic signal; mathematical modeling; optimization; noise level; optimal frequency

\section{INTRODUCTION}

Recent advances in technologies have created many new opportunities to explore underwater resources, which covers about $70 \%$ of the planet earth. Unlike terrestrial wireless sensor networks that rely on radio waves for data exchange, UWSN needs a different approach with far more challenges. Wireless communication in an underwater environment can depend on acoustic waves or optical signals to form a communication network. Like terrestrial WSN, a UWSN is a wireless sensor network that works in an underwater environment to collect data, e.g., temperature, pressure, conductivity, turbidity, and dissolved pollutants seldom to provide some control over submerged devices. The main goal is to collect data precisely in a time-efficient and energy-efficient manner and transmit them to a sink node. The only difference, and challenge at the same time, is that RF signals do not work in an underwater environment, requiring the use of another type of signals to transmit data, namely, acoustic signals to provide wireless connectivity.

Underwater Wireless Sensor Networks has many practical applications. In [3], the authors provided a survey on underwater acoustic sensor network applications that have been suggested and studied in the literature for monitoring and controlling. Authors in [4] reviewed recent applications of UWSNs and discussed possible challenges on the implementation of UWSNs. A comprehensive survey is provided in the latest developments in UWSN in [5]. The underwater applications can be classified into five main classes: environmental monitoring, disaster monitoring, military operations, navigation infrastructure, and sports activities. Many of the challenges and opportunities faced by recent deployments of UWSN were also discussed.

UWSN faces lots of challenges and problems that have been discussed thoroughly in [6] and [8]. They include realtime propagation delay, multipath fading, limited battery, bandwidth constraints of communication channels, and high path loss due to noise. In addition, UWSN Node placement in the third dimension, i.e., depth, significantly affects the transmission path loss and operational energy consumption. As a result, transmission loss is also considerably affected by the characterizes of the water body, such as salinity, temperature, and acidity.

Many commercial low-energy underwater acoustic modem is available nowadays to fit UWSN deployment, such as [2]. Usually, acoustic modems come with a range of acoustic operational frequencies, between $15 \mathrm{kHz}$ and $30 \mathrm{kHz}$. The selection of the optimal transmission frequency should be run-time adjustable during the operation to achieve optimal communication performance.

In this paper, we present a simple and clear mathematical model that can be used as a mathematical basis for an Acoustic Transmission Frequency Optimizer (ATFO) module, as shown in Fig. 1. The AFTO module will read ambient environmental conditions such as temperature and wind speed from its sensor readings; It will then compute the optimized transmission acoustic frequency based on the mathematical model that will be described later. We assume that the direct sink Node will be responsible for setting the transmission frequency, share it, and synchronize operation with all other nodes. This paper will only focus on how to select the optimal frequency. Although many articles in the literature provided similar mathematical modeling, this paper offers a cleaner version with a shareable source code provided for other researchers to utilize.

In this paper, we assume that a UWSN is being deployed for an arbitrary underwater application, as mentioned in previous surveys. We consider a two-dimensional flat network meaning that all nodes in this network are approximately placed in a plain, including the direct sink node, as shown in Fig. 2. We also assume that the direct sensor node is the only node connected with the ground sensor sink node on the sea level. Based on the application and the deployment environment, the depth of the network is decided during the operation. We assume a fixed network setup i.e., no mobile 


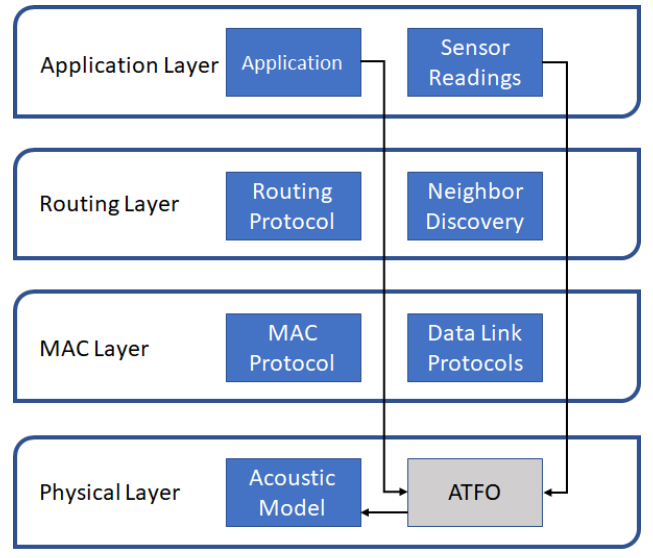

Fig. 1. Underwater Sensor Nodes Networking Layers with AFTO Module

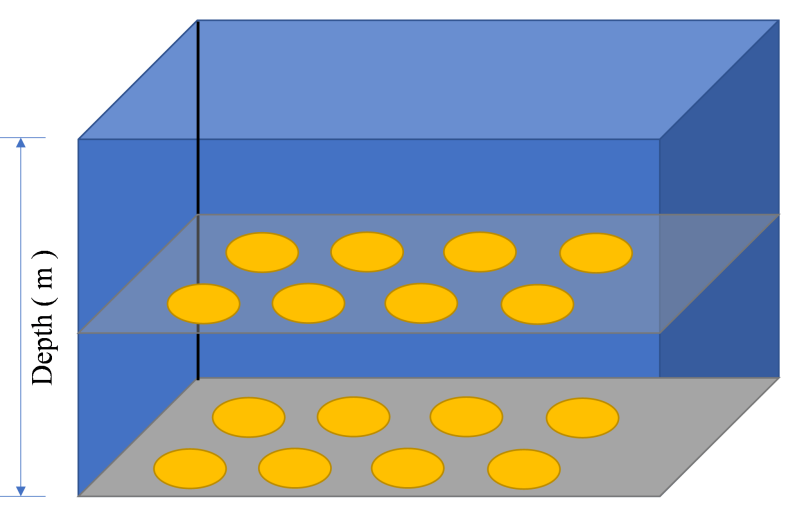

Fig. 2. UWSN Deployment at Various Depths

nodes are considered. We assume that the user can adjust the frequency of the acoustic modems used in UWSN nodes.

\section{RELATED WORK}

Mathematical modeling of the acoustic channel in underwater communication has been studied widely in the literature. Sozer [19] provided a comprehensive overview of many aspects of Underwater Acoustic Networks, including a summarized form of the mathematical modeling part. In [20], a comprehensive tutorial about channel characterization and properties were introduced, including a detailed graph of noise factors affecting link quality. Another higher view of the mathematical modeling concepts of acoustic channels was introduced by [1].

Previous papers showed that transmission loss in underwater communication systems consists of two main parts Absorption due to water body and noise due to external noise factors. Over the years, three main approximations for the absorption coefficient were introduced, namely Fisher [14], Ainslie [13], and Thorp's [12], which have been used in most underwater acoustic channel modeling literature. Noise sources were also characterized and simplified in many articles in the literature, such as [1], [20]. The paper [21] provided an experimental study to analyze noise factors affecting underwater channels.
Developed mathematical models in the literature have been utilized for different purposes. The authors in [7] have provided a detailed mathematical analysis to find the relation between ambient water conditions and transmission loss. In [9] and [10], the authors provided insights about energy-delay and the energy-hops tradeoff in UWSN. In [18], the energy consumption analysis was provided using mathematical models. The distortion analysis of interference or hindrance from other sensors in the network was evaluated by [11].

The author of [15] used the acoustic channel modeling to find out the relation between link capacity and distance. In [16], the authors provided a detailed analysis of noise affecting the underwater communication profile. While in [17], the combined effect of depth and temperature on available capacity was studied.

One concern about most of the mathematical modelings efforts presented in the literature is ambiguity in certain points of the flow. In particular, we found it very difficult to regenerate similar graphs presented in some papers. We can summarize the causes of this problem into the following points:

- Different approximations for certain parameters. For example, in the literature, there are at least three different approximations for the absorption coefficient that are sometimes being used without proper addressing or referencing, making it very difficult for new researchers to know the difference.

- Importance of Units and Scale. Some equations require input parameters to be in certain units (K Meters vs. Meters), and different scales (Log vs. Linear), which are also, sometimes not very clearly mentioned in the model presented.

- Source Code Availability. Authors of literature assume that new researchers can easily construct or build a direct implementation of the mathematical models presented. These simple tasks took a fairly long time due to the first two points than expected to code and to regenerate similar graphs presented in the literature.

In this paper, we did our best to avoid these concerns. We have provided a clear and concise step-by-step model flow. A table listing all parameters with proper unit and scale is provided. Finally, the source code of the developed model, along with generating graphs, is available in [22].

\section{Acoustic Channel Modeling}

Practically, it is very well understood that the underwater Acoustic channel is a very challenging media to establish any communication. These challenges can be summarized as follows:

- Bandwidth Limitation: Acoustic signals operate at very low frequency, limiting the available communication band to a minimum. Typical underwater acoustic hydrophone or modems operates in the range of $15 \mathrm{kHz}$ to $40 \mathrm{kHz}$ [2].

- $\quad$ Noise Level and Sources, there are multiple sources of noise in water bodies that degrades the quality of the acoustic signal. Noise intensity measured in Power 


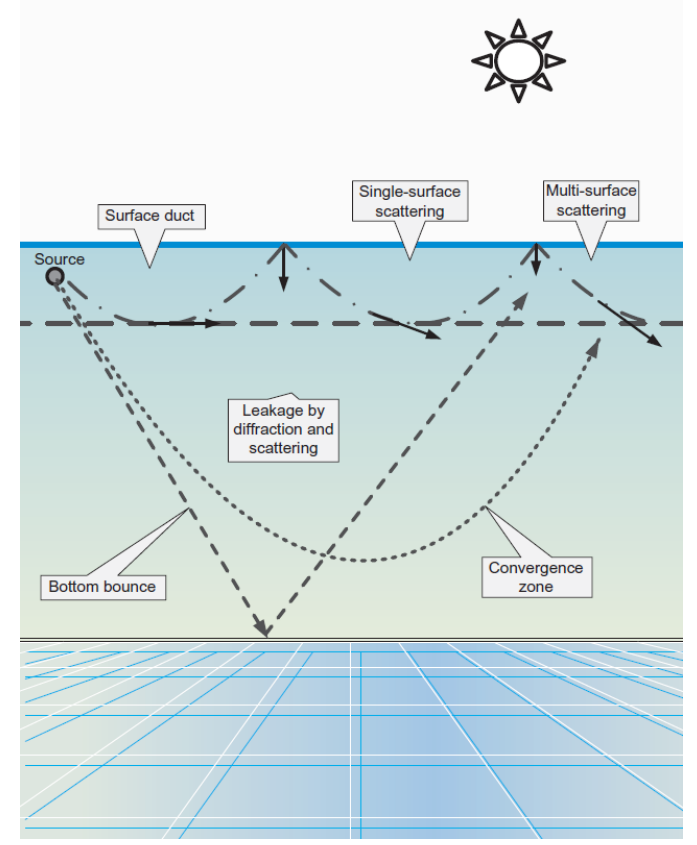

Fig. 3. Acoustic Signal Reflection and Bending Formation from [1]

Spectrum Density with unit $d B$ relative to micro Pascal degrades as frequency increases, as shown in Fig. 5.

- Acoustic Signal Speed and Propagation, Acoustic signals are very slow, $1500 \mathrm{~m} / \mathrm{s}$. This fact emphasizes the propagation delay, which is usually neglected in the case of a terrestrial wireless network. The high propagation delay also magnifies the multipath problem of acoustic signal radiation. Also, acoustic signals in water bodies have a special form of bending and refraction, shown in Fig. 3, making the multipath problem even more challenging.

- Attenuation Level, Water bodies have more mass than air, making signal propagation through that body more difficult. Acoustic signal suffers from spreading and absorption in the water body. As a rule of thumb and as shown in Fig. 4, attenuation levels increases as frequency increases.

- Affecting parameters; although the frequency is the dominant factor for underwater acoustic signal prorogation, it still suffers from multiple other factors that have a complicated combined effect. A summarized list of all factors is shown in Table I.

To establish a wireless communication link between two nodes, the received power at the destination node should be higher than a certain threshold called $r x$ Sensitivity Level $r x_{\text {Level }}$. This rule is true regardless of channel and type of carrier wave, i.e., RF vs. Acoustic. Mathematically, this condition can be formulated as

$$
r x_{\text {power }} \geq r x_{\text {level }}
$$

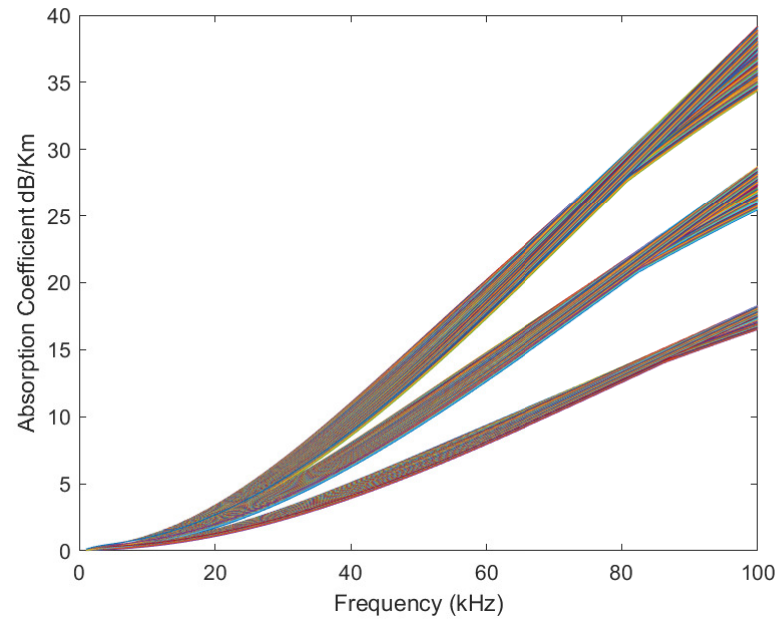

Fig. 4. Absorption Coefficient, $\alpha$ [dB/km] for Different Combination of Input Variables as shown in Table II

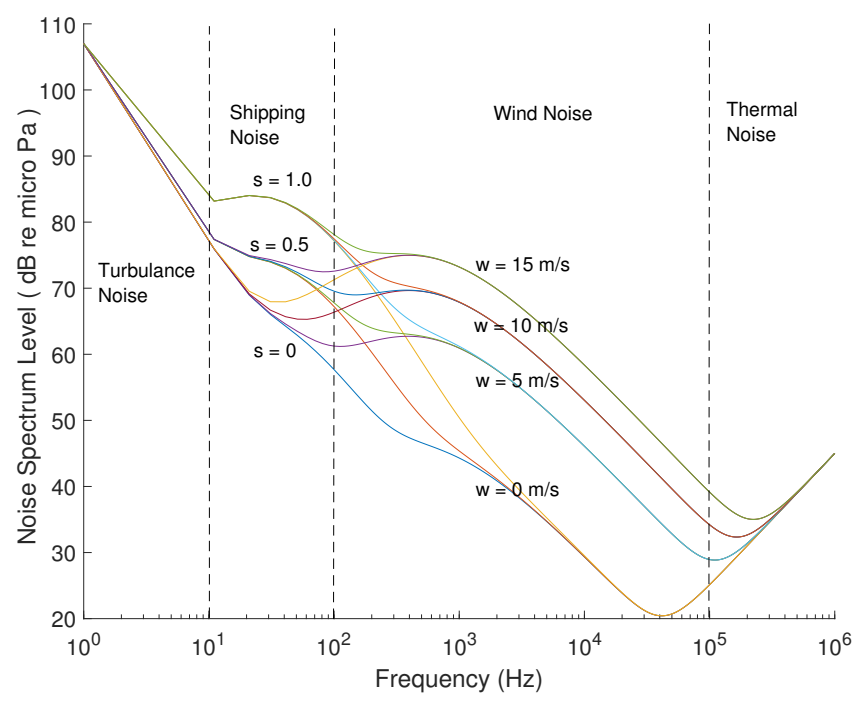

Fig. 5. Noise Loss Spectrum Level (dB re $1 \mu$ Pascal)

where $r x_{\text {power }}$ is the reception power level measured at the destination node. Using $d B$ to simplify calculations, $r x_{\text {power }}$ can be calculated as

$$
r x_{\text {power }}=t x_{\text {power }}-A(f)-N(f)
$$

Where $A(f)$ is the signal loss due to attenuation, and $N(f)$ is the loss due to Noise. The signal attenuation loss $A(f)$ in $d B$ given in Equation $3^{1}$ composed of two losses namely, spreading and absorption. The spreading loss is due to the geometric spreading of signal propagation it is a function of transmission range $r$ and the spreading factor $\kappa$.For our

\footnotetext{
${ }^{1}$ One should note the scale and units of parameters plugged into such equations, please refer to table I
} 


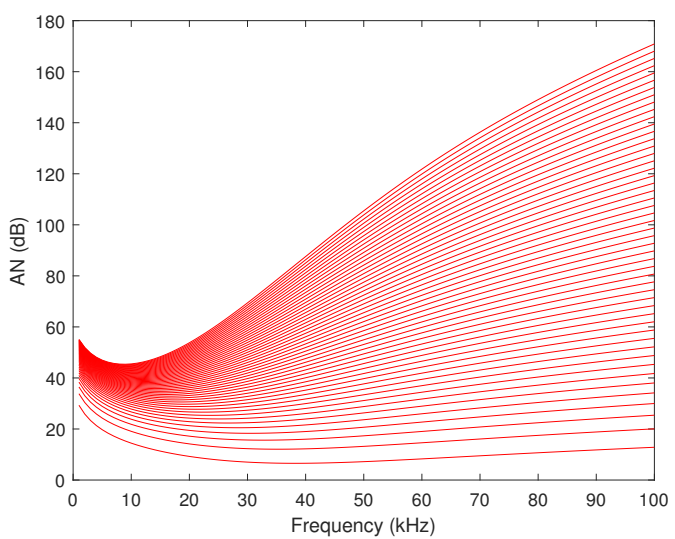

(a) Effect of Different Transmission Ranges on AN

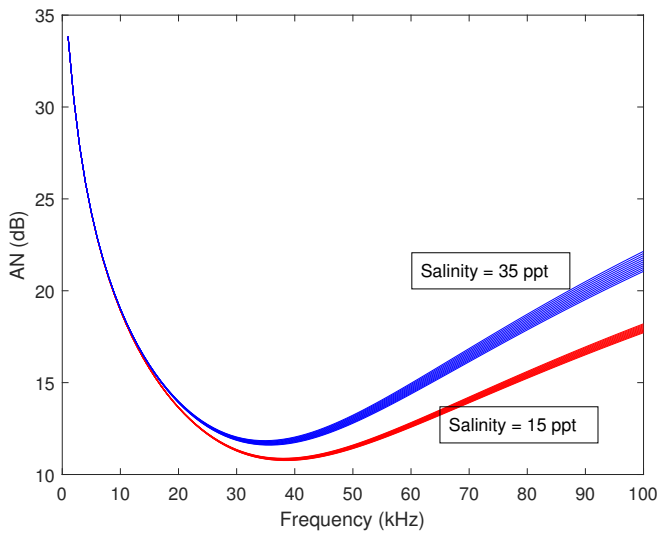

(c) Effect of Different Salinity Levels on AN

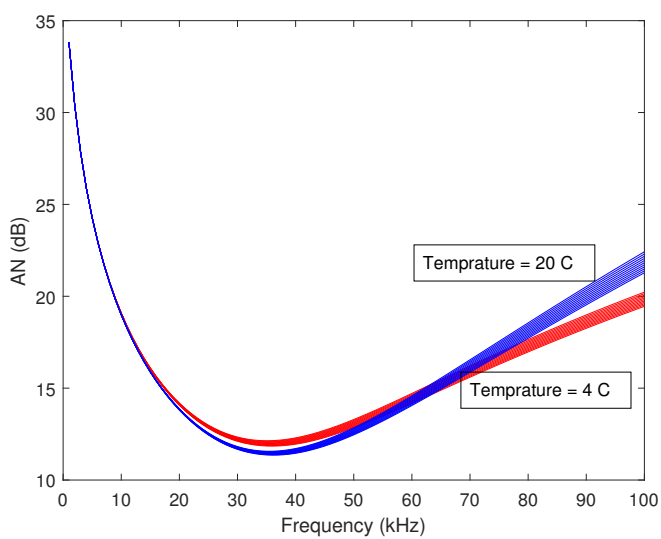

(b) Effect of Different Temprature Values on AN

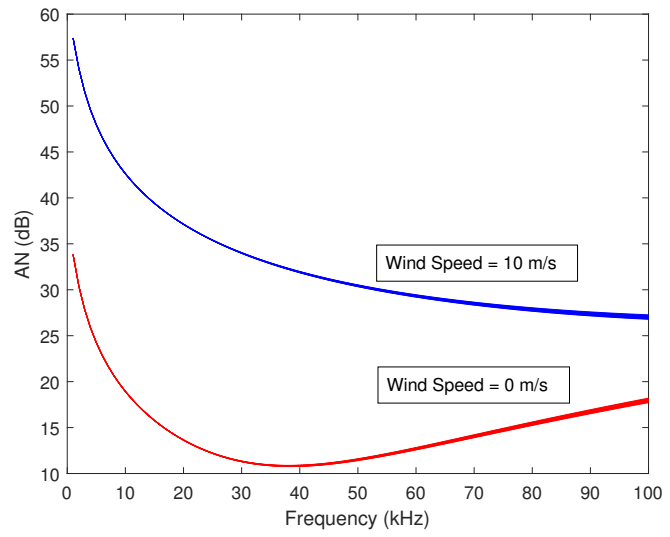

(d) Effect of Different Wind Speed Values on AN

Fig. 6. Effect of Different Single Parameter Values on AN

calculations and graphs in this paper, we will always use $\kappa=1.5$.

$$
10 \log A(f)=\kappa .10 \log r+r .10 \log \alpha(f)
$$

The absorption loss is a function of transmission range $r$ and absorption coefficient $\alpha$, which describes water body capability to absorb the energy from the acoustic signal and convert it into heat. A higher absorption coefficient means a higher $\mathrm{dB}$ loss from the acoustic signal. The absorption coefficient $\alpha$ value is dominated by frequency but also temperature, $\mathrm{pH}$ level, depth level, water salinity can affect its value. There are many models that approximate the absorption coefficient empirically, such as Thorp's model and Fisher models [12], [14]. However, in this paper, we will use an approximation suggested by Ainslie and McColm [13] presented in the following formula

$$
\alpha=\gamma_{1} \frac{f_{1} f^{2}}{f_{1}^{2}+f^{2}}+\gamma_{2} \frac{f_{2} f^{2}}{f_{2}^{2}+f^{2}}+\gamma_{3} f^{2}
$$

where,

$$
\begin{aligned}
f_{1} & =0.78 \sqrt{\frac{s}{35}} \exp ^{\frac{t}{26}}, \\
f_{2} & =42 \exp ^{\frac{t}{17}} \\
\gamma_{1} & =0.106 \exp ^{\frac{p H-8}{0.56}} \\
\gamma_{2} & =0.52\left(1+\frac{t}{43}\right)\left(\frac{s}{35}\right) \exp ^{\frac{-d}{6}} \\
\gamma_{3} & =0.00049 \exp ^{-\left(\frac{t}{27}+\frac{d}{17}\right)}
\end{aligned}
$$

For correct implementation of all equations, it is very important to understand and know units for all parameters which are summarized in Table I.

We have implemented the attenuation loss approximation above and calculated the resulted absorption coefficient for many possible combinations of input variables shown in Fig. 4. Note the increasing trend of the absorption coefficient with increasing frequency.

The Noise Loss in 2 is mainly due to ambient noise. There are four major sources for ambient noise in underwater acoustic channel namely; turbulence, shipping, wind driven waves and thermal noise. Noise is measured as power spectral 


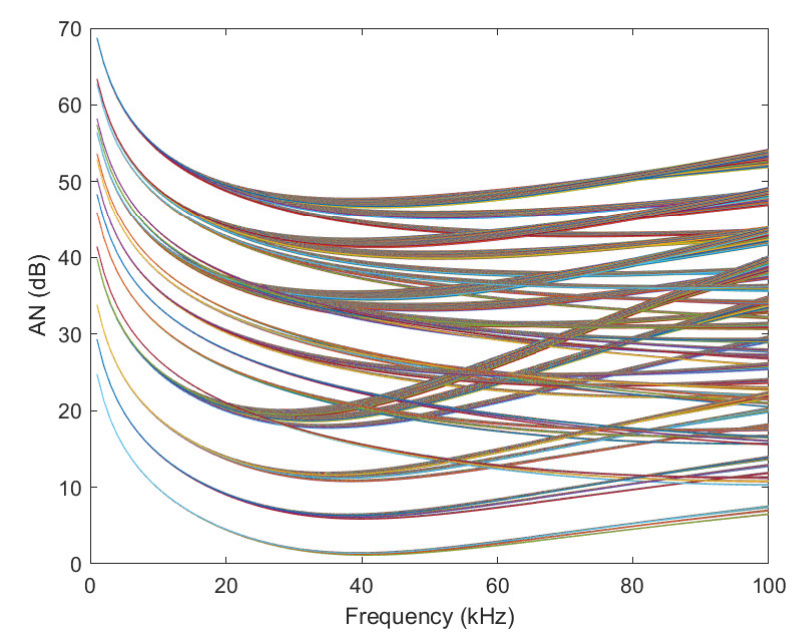

Fig. 7. Absorption Coefficient, $\alpha$ [dB/km] for Different Combination of Input Variables as shown in Table II

TABLE I. Acoustic Channel Model Parameters And Units

\begin{tabular}{|c|c|c|}
\hline Parameter & Description & Unit \\
\hline$r x_{\text {power }}$ & Received Signal Power & $\mathrm{dB}$ \\
\hline$r x_{\text {level }}$ & Received Signal Threshold & $\mathrm{dB}$ \\
\hline$t x_{\text {power }}$ & Transmitting Signal Power & $\mathrm{dB}$ \\
\hline$A$ & Attenuation Loss & $\mathrm{dB}$ \\
\hline$N$ & Noise Loss & $\mathrm{dB}$ \\
\hline$r$ & Communication Range & $\mathrm{Km}$ \\
\hline$f$ & Frequency & $\mathrm{kHz}$ \\
\hline$t$ & Water Body Temperature & ${ }^{\circ}$ Celsius \\
\hline$d$ & Water Depth & $\mathrm{Km}$ \\
\hline$s$ & Water Salinity & $\mathrm{ppt}$ \\
\hline$p H$ & Water Acidity Level & \\
\hline$w$ & Sea Surface Wind Speed & $\mathrm{m} / \mathrm{s}$ \\
\hline$s h$ & Shipping Activity Factor & \\
\hline$\kappa$ & Spreading Coefficient & \\
\hline
\end{tabular}

density and its unit is $d B$ relative to $\mu$ Pascal. Noise can be approximated as given by the following formula:

$$
N=N_{t}+N_{s h}+N_{t h}+N_{w}
$$

where $N_{t}, N_{s h}, N_{t h}$ and $N_{w}$ are given by the following formulas:

$$
\begin{aligned}
& 10 \log N_{t}=17-30 \log (f) \\
& 10 \log N_{s h}=40+20(\operatorname{sh}-0.5)+26 \log (f)-60 \log (f+0.03) \\
& 10 \log N_{w}=50+7.5 w^{1 / 2}+20 \log (f)-40 \log (f+0.4) \\
& 10 \log N_{t h}=-15+20 \log (f)
\end{aligned}
$$

where $s h$ and $w$ are Shipping Activity Factor and Wind Speed, respectively.

Each noise source affects a particular range of frequencies. Low-frequency region, $f<10 \mathrm{~Hz}$ is influenced by turbulence noise. The frequency range of $10 \mathrm{~Hz}-100 \mathrm{~Hz}$ is majorly influenced by shipping activity factor $s h$, whose value ranges between 0 and 1 for low and high activity. Wind-driven waves cause surface motion, which is the dominant factor of noise in the frequency region $100 \mathrm{~Hz}$ to $100 \mathrm{kHz}$. It is measured in $\mathrm{m} / \mathrm{s}$, and this frequency operating region is used by the majority of acoustic systems. Thermal noise contributes for $f>100 \mathrm{kHz}$ [15]. We have implemented Equations 5 and 6 for different values of $s h$ and $w$ over the frequency spectrum [1 Hz to $100000 \mathrm{~Hz}$ ]. Fig. 5 shows the Noise levels in different spectral regions with the dominant factors in each region. One can notice the decrease trending line as the frequency increases, which shows an opposite behavior compared to attenuation loss above.

Combining both losses effects, i.e., Noise and Attenuation, in the product form, $A N$ would give us insight about communication quality for different sets of conditions. $A N$ is the total loss incurred by the acoustic signal, which in $d B$ can be expressed as in equation 2. Now, let us first examine the single effect of different parameters on $A N$, as shown in Fig. 6. We run the mathematical model extensively using the parameter combinations listed in Table $\mathrm{II}^{2}$.

Fig. 6(a) shows the effect of different communication ranges while fixing all other parameters. You can notice the rabid increase of $A N$ product as the transmission range increases especially with higher frequency. In (b), the increase in the temperature slightly increases the loss value as the frequency increases. Salinity level changes affect $A N$, as shown in (c), which is also has a limited effect. The major effect happens in Figure (d) with wind speed. As wind speed increases from $0 \mathrm{~m} / \mathrm{s}$ to $10 \mathrm{~m} / \mathrm{s}, A N$ increases up to three times.

Fig. 6 shows three main observations as follows:

- Different parameters used in the acoustic channel model have different effects on the $A N$ product.

- Noise loss has two different trending effects as frequency increases with various affecting factors in each frequency range. In general, the loss due to noise decreases as frequency increases, but at the same time, the loss due to attenuation increases as frequency increases.

- The contradicting trends of both losses create a minimal turning point where $A N$ is the minimum. The frequency that generates that minimal $\mathrm{AN}$ value is the

\footnotetext{
${ }^{2}$ The implementation of the mathematical model is available at https://emadfelemban.org/coralsense
} 


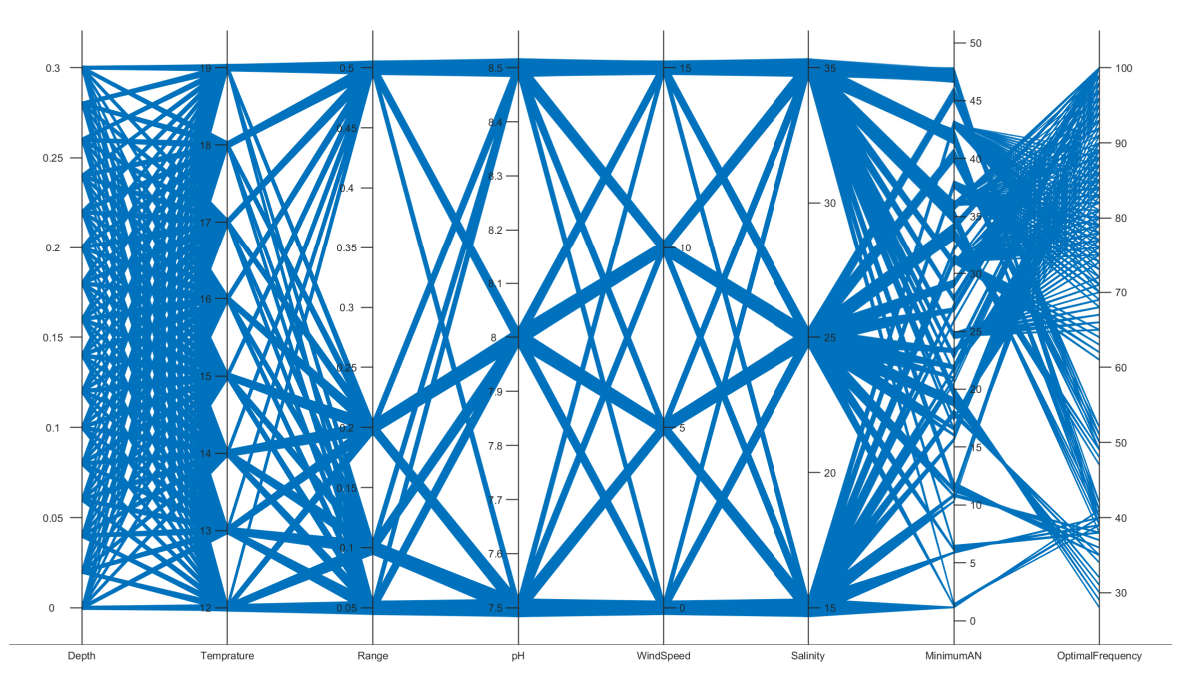

Fig. 8. A Parallel Coordinate Plot Showing All Combinations of Parameter Values Selected

optimal frequency. This optimal frequency changes as the conditions and requirements change.

- Among all parameters that are used in the acoustic channel model, wind speed has a very strong effect on $A N$.

Fig. 7 shows all the curves for all different combinations to get a comprehensive view. Fig. 8 is a parallel coordinate graph that shows the parameters and their values used to create Fig. 7. The same figure shows the different various optimal frequencies for each case.

\section{Optimal Selection of Communication PARAMETERS}

The quality of underwater acoustic communication depends on multiple parameters that can be categorized into three categories:

- Environmental parameters that are related to the ambient environment conditions around the communication area such as temperature, salinity level, wind speed, shipping factor.

- Deployment parameters that are related to deployment conditions of the network such as depth level and transmission range between nodes. Note that these parameters can be changed either manually in the case of fixed networks or using mobile capabilities in the case of mobile or ROV network.

- Communication Parameters that can change and affect the communication channel performance between the source and destination nodes such as transmission power and frequency. Most of the commercially available underwater acoustic modems provide flexibility in setting frequency and transmission power and changing them by software.
Fig. 9 shows different $A N$ vs Frequency curves calculated with different combinations of parameters. Using the same data, we find the optimal frequency for each case and plot Fig. 6. The optimal transmission frequency provides the lowest AN value and thus most likely provides the best performance in the communication channel. For all plots, we changed the wind speed from $0 \mathrm{~m} / \mathrm{s}$ to $15 \mathrm{~m} / \mathrm{s}$ and plotted four curves for each parameter. Fig. 6(a) shows the optimal frequencies as depth changes from $0 \mathrm{Km}$ to $10 \mathrm{Km}$ vs. the increase of wind speed. In Fig. 6(b), (c) and (d) we varied the transmission range, temperature and salinity levels. It is very clear that we need to have a dynamic way to select the appropriate transmission frequency to establish a good communication channel.

\section{CONClusion}

This paper provides a gateway to find the optimum communication parameters for underwater communication. It offers an insight into the relationship between the different parameters that govern the underwater acoustic communication channel. We have reviewed many mathematical models available for underwater acoustic communication. We implemented our own version of the model and made it available online. We ran many input parameter combinations by changing depth, temperature, wind speed salinity to measure the effect on path loss. We found that wind speed has the most impact on path loss. Finally, we shed some light on optimizing the communication parameters, specifically the frequency. Our future work will include developing the frequency optimizer module and run experimental simulation scenarios to measure the effect of optimal frequency in the simulation environment.

\section{ACKNOWLEDGMENT}

This work is supported by the National Science, Technology and Innovation program NSTIP Grante number "11INF1688-10" by King Abdulaziz City of Science and Technology. 


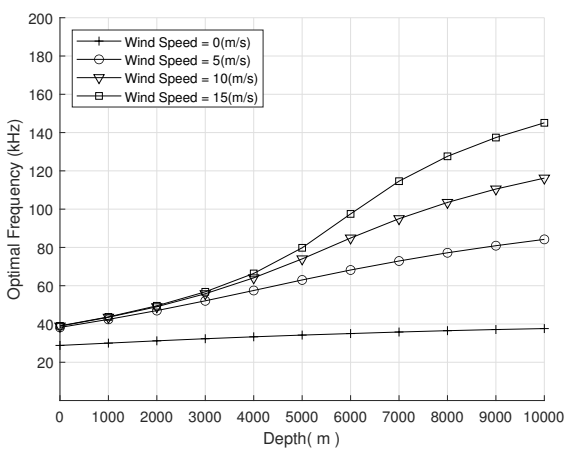

(a) Optimal frequency for various depths

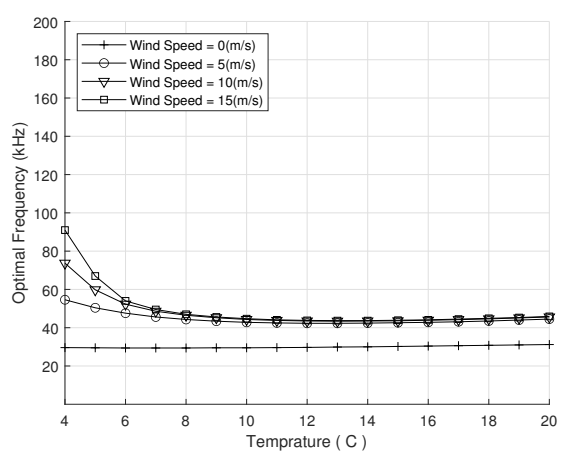

(c) Optimal frequency for various temperatures

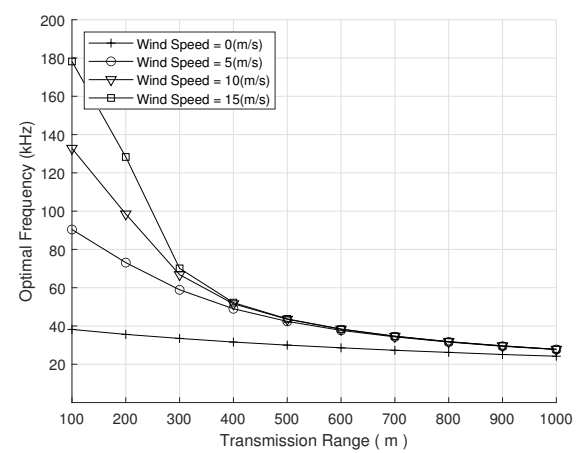

(b) Optimal frequency for various distances

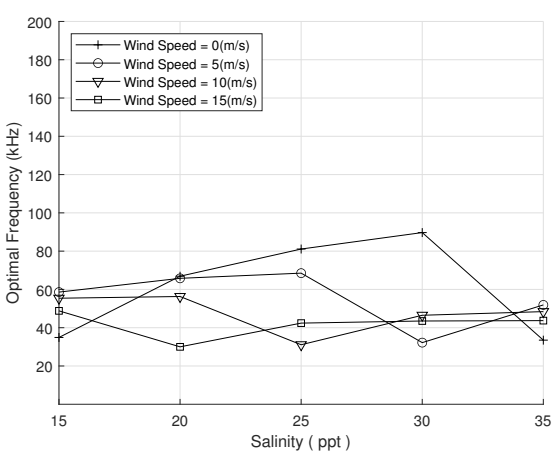

(d) Optimal frequency for various Salinity Levels

Fig. 9. Optimal Frequency Graphs while Changing Different Parameters

\section{REFERENCES}

[1] R. Christ and R. Wemli, "The ROV Manual”, 2nd Edition, Elsevier 2014

[2] https://popotomodem.com/

[3] M. Murad, A. Sheikh, M. Manzoor, E. Felemban, and S. Qaisar, "A Survey on Current Underwater Acoustic Sensor Network Applications". International Journal of Computer Theory and Engineering, 2015

[4] P. Amoli, "An Overview on Current Researches on Underwater Sensor Networks: Applications, Challenges and Future Trends", International Journal of Electrical and Computer Engineering, vol. 6, p. 955, 2016.

[5] E. Felemban, F. Shaikh, U. Qureshi, A. Sheikh, S. Qaisar, "Underwater sensor network applications: A comprehensive survey", International Journal of Distributed Sensor Networks, vol. 2015, pp. 1-14, Aug. 2015.

[6] H. Jindal, S. Saxena and S. Singh, "Challenges and issues in underwater acoustics sensor networks: A review", International Conference on Parallel, Distributed and Grid Computing, 2014.

[7] S. Kohli and P. Bhattacharya, "Characterization of Acoustic Channel for Underwater Wireless Sensor Networks", Annual IEEE India Conference (INDICON), pp. 1-4, 2005.

[8] I. Akyildiz, D. Pompili and T. Melodia, "Underwater acoustic sensor networks: Research challenges", Ad Hoc Networks, vol. 3, pp. 257-279, 2005.

[9] X. Jin, Y. Chen and X. Xu, The analysis of hops for multi-hop cooperation in Underwater Acoustic Sensor Networks, 2016 IEEE/OES China Ocean Acoustics (COA), Harbin, 2016, pp. 1-5.

[10] M. Felemban and E. Felemban, "Energy-delay tradeoffs for Underwater Acoustic Sensor Networks", "First International Black Sea Conference on Communications and Networking (BlackSeaCom)", Batumi, pp. 4549. 2013.

[11] A. Stefanov, "Distortion analysis of underwater acoustic sensor net- works", 7th International Conference on New Technologies, Mobility and Security (NTMS)", pp. 1-4, 2015.

[12] L. Berkhovskikh and Y.Lysanov, "Fundamentals of Ocean Acoustics" New York: Springer, 1982.

[13] M. Ainslie and J. McColm, "A simplified formula for viscous and chemical absorption in sea water," Acoustical Society of America Journal, vol. 103, pp. 1671-1672, Mar. 1998.

[14] F. Fisher and V. Simmons, "Sound Absorbtion in sea water" Acoustical Society of America Journal, vol.62, pp. 558-564, 1977.

[15] M. Stojanovic, "On the relationship between capacity and distance in an underwater acoustic communication channel," ACM SIGMOBILE Mobile Computing and Communications Review, vol. 11, no. 4, pp. 34-43, 2007

[16] K. Mackenzie, "Nineterm equation for sound speed in the oceans," The Journal of the Acoustical Society of America, vol. 70, p. 807, 1981.

[17] A. Sehgal, I. Tumar, and J. Schonwalder, "Variability of Available Capacity due to the Effects of Depth and Temperature in the Underwater Acoustic Communication Channel", IEEE Ocean's, pp. 1-6, 2009.

[18] A. Sehgal, C. David and J. Schonwalder, "Energy consumption analysis of underwater acoustic sensor networks," IEEE OCEANS'1, pp. 1-6, 2009.

[19] E. Sozer, M. Stojanovic, and J. Proakis, "Underwater acoustic networks," IEEE Journal of Oceanic Engineering, vol. 25, no. I, pp. 72-83, January 2000.

[20] M. Stojanovic, J. Preisig, "Underwater Acoustic Communication Channels: Propagation Models and Statistical Characterization," IEEE Communications Magazine, January 2009.

[21] C. Bassett, J. Thomson, B. Polague, "Characteristics of Underwater Ambient Noise at a Proposed Tidal Energy Site in Puget Sound," Oceans 2010, September 2010.

[22] https://emadfelemban.org/coralsense 